\title{
Simulation of freight trains equipped with partially filled tank containers and related resonance phenomenon
}

\section{Vera, J. Paulin, B. Suarez and M. Gutierrez}

In order to study the fluid motion - vehicle dynamics interaction, a model of four liquid filled two-axle container freight wagons was set up. The railway vehicle has been modelled as a multibody system. To include fluid sloshing, an equivalent mechanical model has been developed and incorporated. The influence of several factors has been studied in computer simulations, such as track defects, curve negotiation, train velocity, wheel wear, liquid and solid wagonload, and container baffles. SIMPACK has been used for multibody systems analysis, and ANSYS for liquid sloshing modelling and equivalent mechanical systems validation. Acceleration and braking manoeuvres of the freight train set the liquid cargo into motion. This longitudinal sloshing motion of the fluid cargo inside the tanks initiated a swinging motion of some components of the coupling gear. The coupling gear consists of UIC standard traction hooks and coupling screws that are located between buffers. One of the coupling screws is placed in the traction hook of the opposite wagon thus joining the two wagons, whereas the un-used coupling screw rests on a hanger. Simulation results showed that, for certain combinations of type of liquid, filling level and container dimensions, the liquid cargo could provoke an undesirable, although not hazardous, release of the un-used coupling screw from its hanger. The coupling screw's release was especially obtained when a period of acceleration was followed by an abrupt braking manoeuvre at $1 \mathrm{~m} / \mathrm{s} 2$. It was shown that a resonance effect between the liquid's oscillation and the coupling screw's rotary motion could be the reason for the coupling screw's undesired release. Possible solutions in order to avoid the phenomenon are given. 


\title{
Simulation of freight trains equipped with partially filled tank containers and related resonance phenomenon
}

\author{
C. Vera ${ }^{1}$, J. Paulin ${ }^{2}$, B. Suarez ${ }^{2 *}$ and M. Gutierrez ${ }^{3}$ \\ ${ }^{1}$ Department of Mechanical Engineering, Polytechnic University of Madrid (UPM), Spain \\ ${ }^{2}$ Railway Technology Research Centre (CITEF), Madrid, Spain \\ ${ }^{3}$ UN Mercancías, RENFE Operadora, Madrid, Spain \\ * Corresponding author: CITEF, C/ José Gutiérrez Abascal n² 2, ES-28006 Madrid, Spain, email: \\ citef.bsuarez@etsii.upm.es
}

\begin{abstract}
In order to study the fluid motion - vehicle dynamics interaction, a model of four liquid filled two-axle container freight wagons was set up. The railway vehicle has been modelled as a multibody system. To include fluid sloshing, an equivalent mechanical model has been developed and incorporated. The influence of several factors has been studied in computer simulations, such as track defects, curve negotiation, train velocity, wheel wear, liquid and solid wagonload, and container baffles. SIMPACK has been used for multibody systems analysis, and ANSYS for liquid sloshing modelling and equivalent mechanical systems validation.

Acceleration and braking manoeuvres of the freight train set the liquid cargo into motion. This longitudinal sloshing motion of the fluid cargo inside the tanks initiated a swinging motion of some components of the coupling gear. The coupling gear consists of UIC standard traction hooks and coupling screws that are located between buffers. One of the coupling screws is placed in the traction hook of the opposite wagon thus joining the two wagons, whereas the un-used coupling screw rests on a hanger.

Simulation results showed that, for certain combinations of type of liquid, filling level and container dimensions, the liquid cargo could provoke an undesirable, although not hazardous, release of the un-used coupling screw from its hanger. The coupling screw's release was especially obtained when a period of acceleration was followed by an abrupt braking manoeuvre at $1 \mathrm{~m} / \mathrm{s}^{2}$. It was shown that a resonance effect between the liquid's oscillation and the coupling screw's rotary motion could be the reason for the coupling screw's undesired release. Possible solutions in order to avoid the phenomenon are given.
\end{abstract}

Keywords: screw couplings, freight wagons, liquid cargo, sloshing, resonance 


\section{NOTATION}

$b \quad[\mathrm{~m}] \quad$ width of the rectangular tank

$f_{\text {liquid }}[\mathrm{Hz}]$ first eigen frequency of the liquid oscillating longitudinally in the tank container

$f_{\text {screw }}[\mathrm{Hz}]$ first eigen frequency of the rotating motion of the coupling screw about its attachment point

$g \quad\left[\mathrm{~m} / \mathrm{s}^{2}\right]$ gravitational acceleration

$h \quad[\mathrm{~m}] \quad$ height of the fluid's free surface

$h_{0} \quad[\mathrm{~m}] \quad$ vertical distance between the fixed point mass, $m_{0}$, and the tank's floor

$k_{1} \quad[\mathrm{~N} / \mathrm{m}]$ total spring stiffness

$l_{l} \quad[\mathrm{~m}] \quad$ vertical distance of the oscillating mass, $m_{l}$, from the fluid's free surface

$l_{2} \quad[\mathrm{~m}] \quad$ vertical distance of the fixed mass, $m_{o}$, from the fluid's free surface

$l_{p} \quad[\mathrm{~m}] \quad$ length of pendulum

$m_{0} \quad[\mathrm{~kg}] \quad$ fixed mass

$m_{l} \quad[\mathrm{~kg}] \quad$ oscillating mass

$m_{o} \quad[\mathrm{~kg}] \quad$ point mass fixed to the tank representing the stationary part of the liquid

$m_{p} \quad[\mathrm{~kg}] \quad$ point mass attached at the pendulum's free end, representing the fraction of the liquid that takes part in the oscillating motion

$m_{t} \quad[\mathrm{~kg}] \quad$ total fluid mass

$Q_{0} \quad[\mathrm{~N}] \quad$ static vertical wheel load

$R \quad[\mathrm{~m}] \quad$ curve radius

$t \quad[\mathrm{~s}] \quad$ simulation time of SIMPACK model

$v \quad[\mathrm{~m} / \mathrm{s}] \quad$ train velocity

$w \quad[\mathrm{~m}] \quad$ length of the rectangular tank

$\Delta Q \quad[\mathrm{~N}] \quad$ wheel unload (deviation from $\mathrm{Q}_{0}$ )

$\rho \quad\left[\mathrm{kg} / \mathrm{m}^{3}\right]$ liquid's density

\section{INTRODUCTION}

Computer aided analysis of the dynamic behaviour of train vehicles is an interesting alternative to prototype testing and real experiments, as, in general, it brings along an important cost reduction. Dynamic computer simulations of well-adjusted models usually provide results that are very close to those measured in reality and thus serve to take decisions in railway engineering based on computer calculations.

Throughout recent years, the Railway Technology Research Centre, CITEF (Centro de Investigación en Tecnologías Ferroviarias), of the Polytechnic University of Madrid, UPM (Universidad Politécnica de Madrid), has obtained experience in setting up detailed models of railway 
vehicles using the program for multibody systems (MBS) simulation, SIMPACK, and the finite element analysis (FEM) program, ANSYS [1].

The work presented in this paper was the result of a scientific collaboration between CITEF (a research centre within the Polytechnic University of Madrid) and the Spanish Railway Operator (RENFE Operadora).

\subsection{Freight trains equipped with tank containers}

The freight trains that were modelled are typical two-axle wagons carrying tank containers filled with a liquid load up to the maximum fill level allowed. The maximum fill level depends on both maximum axle load and on the vapour pressure resulting from the gaseous phase of the liquid contained within the tanks [2].

When the vehicle starts moving, the fluid contained inside the tanks begins an inherent oscillation. The peculiarity of fluid motion within partially filled closed containers is commonly referred to as "sloshing". It is well known that in almost all kinds of liquid transportation systems, the sloshing superimposes itself upon the vehicle motion and occasionally can lead to critical situations. In case of railway transportation systems, such situations could be the train's derailment or even the roll over of the container wagon when passing through tight curves.

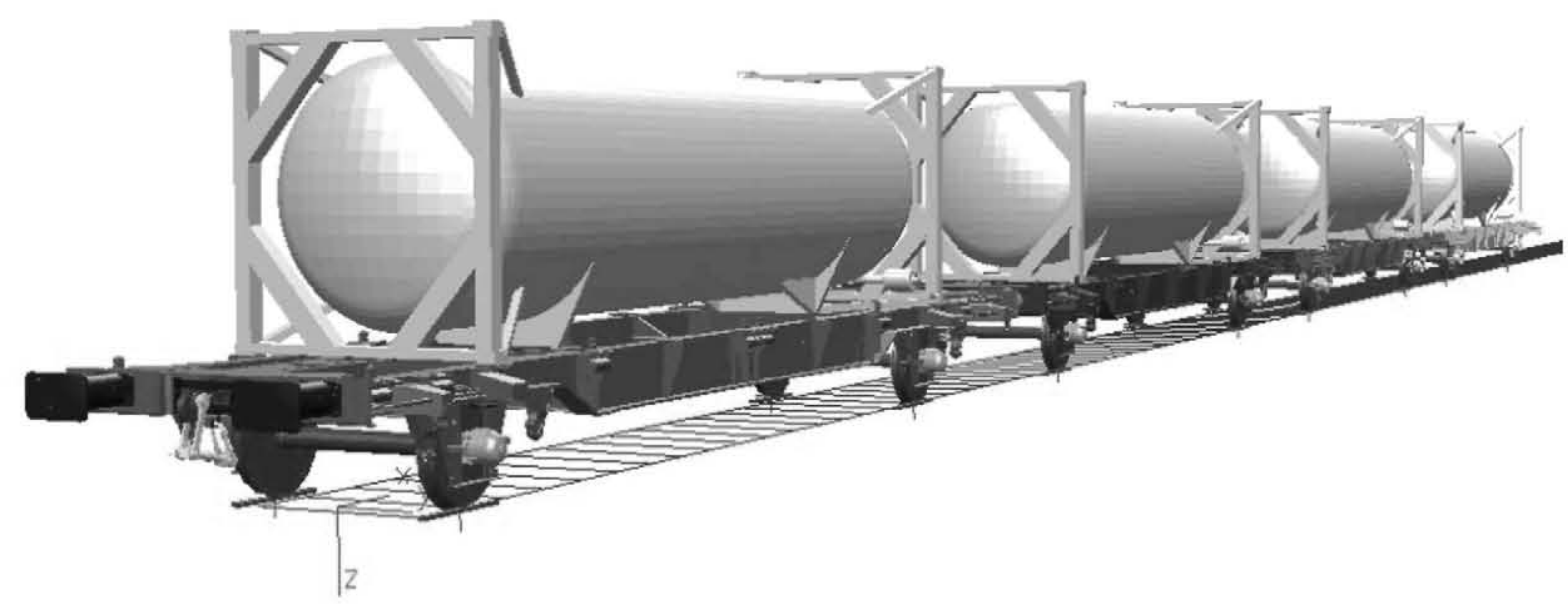

Fig. 1 Freight trains equipped with tank containers

The modelling of freight trains using advanced computer programs apt for simulating multibody systems, such as SIMPACK, is well known [3,4]. However, when it comes to combining and simultaneously computing the multibody system equations and the complex fluid dynamics equations (such as Navier-Stokes), modelling gets complicated and very time consuming.

In the next section the authors will give a description of the vehicle model, followed by an explanation of the incorporation of the liquid load's sloshing motion using simplified one-degree-offreedom models, in section 3 .

\section{DEVELOPMENT OF THE VEHICLE MODEL}

The final vehicle model consisted of compositions of two-axle container wagons that were linked to each other by standard UIC screw couplings that are located between buffers. One wagon consisted of 
the following major components, modelled by means of rigid bodies in the multibody system program, SIMPACK:

- Wheelsets ( 2 bodies)

- Axle boxes ( 4 bodies)

- $\quad$ Platform (1 body)

- Buffers and draw gears (14 bodies)

- $\quad$ Filled tank container ( 2 bodies)

The data necessary for describing the rigid bodies, such as mass, centre of gravity location and moments of inertia were calculated based on three-dimensional CAD drawings or were taken directly from the two-dimensional technical drawings or description sheets of the tank container wagons.

\subsection{Detailed model}

In a first step, a highly detailed model of the train vehicle was set up. Four wagons were included in the train model, since it was opined that four tank container wagons were enough in order to approximate well to real vehicle behaviour. The detailed, four-wagon-model, had a total of 124 rigid bodies, 32 of which were so-called "dummy bodies" that had zero degrees-of-freedom (DOF). The dummy bodies were needed for incorporating substructures or for adding the fixed masses of the fluid model. The detailed multi-body system model had 117 DOF altogether.

By including many details, the results obtained were expected to approximate well to real vehicle behaviour. The detailed model included various features that will be described in the following paragraphs:

\subsubsection{Wheel and rail profiles}

The final model included standard UIC 54 rails with an inclination of 1:20. The standard Spanish broad gauge of $1668 \mathrm{~mm}$ was used. The wheels had a radius of $920 \mathrm{~mm}(840 \mathrm{~mm})$ in their unworn (worn) state. A typical RENFE wheel profile was used for the wheel-rail contact calculation.

\subsubsection{UIC leaf spring suspension}

The UIC leaf spring suspension was modelled using simple springs and dampers in all three directions (longitudinal, lateral, and vertical). A more complex suspension model that would include the entire leaf spring mechanism as well as friction between leafs, was thought about. However, in order to reduced calculation time, the final model only included stiffness and damping in all three directions. The vertical stiffness of the leaf spring suspension was taken from a RENFE data sheet that contained a measured non-linear force-displacement relationship, as shown in Fig. 2. 


\section{Measured vertical leaf spring stiffness}

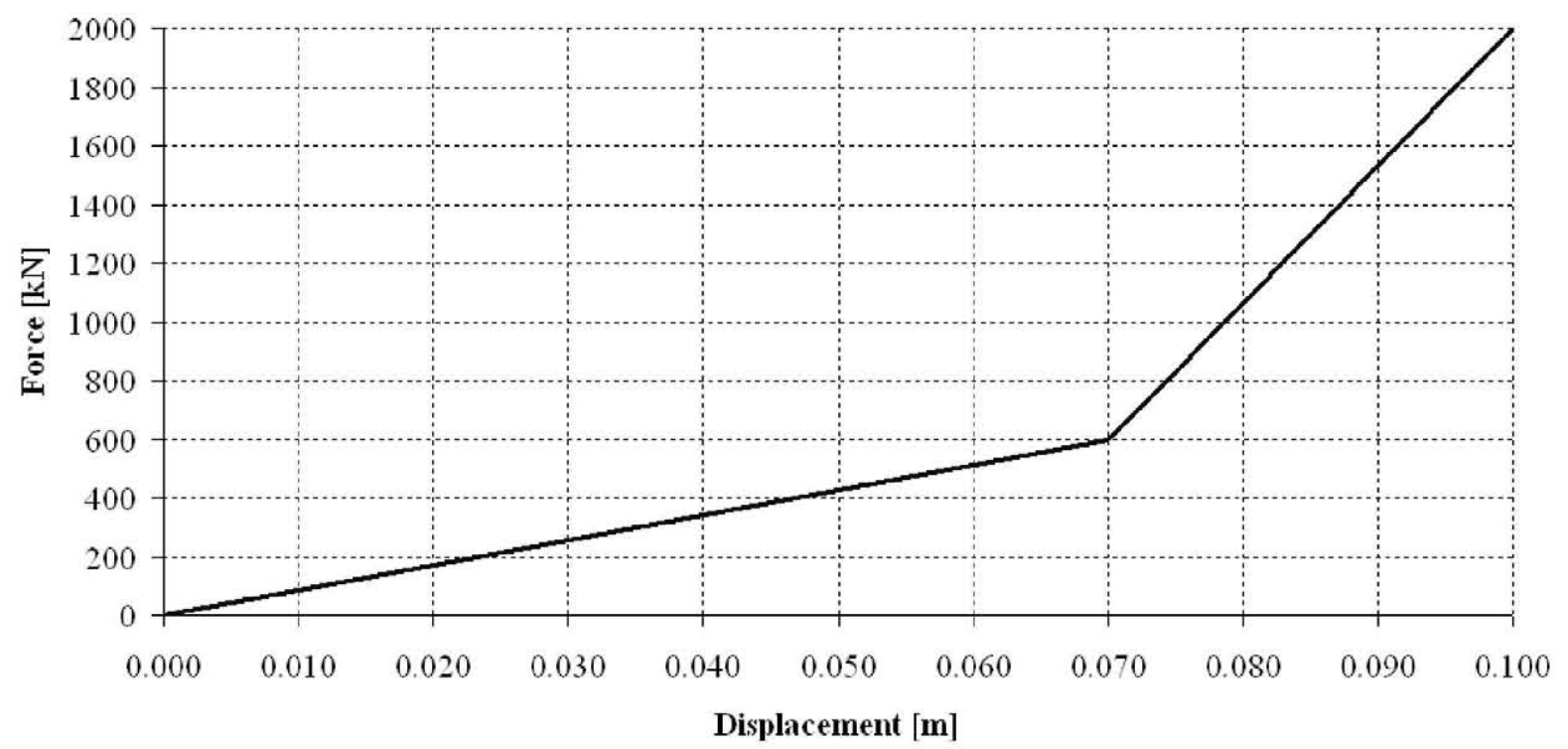

Fig. 2 Input function for the non-linear vertical stiffness of the leaf spring suspension

The values used for damping and for the remaining stiffness were taken from a benchmark in which a very similar two-axle freight wagon was described [4]. Tab. 1 gives a summary of all values used.

\begin{tabular}{|l|c|c|}
\hline \multicolumn{1}{|c|}{ Direction } & Stiffness [N/m] & Damping [Ns/m] \\
\hline Longitudinal (x) & 12000000 & 7000 \\
\hline Lateral $(\mathrm{y})$ & 640000 & 8000 \\
\hline Vertical $(\mathrm{z})$ & As given in Fig. 2 & 1000 \\
\hline
\end{tabular}

Tab. 1 Stiffness and damping of the primary suspension

Both longitudinal, and vertical bump stops were included in the model. The vertical bump stops limit the relative displacement between the platform and the axle boxes to $106 \mathrm{~mm}(35 \mathrm{~mm})$ for the un-loaded (loaded) freight wagon. The longitudinal bump stop limits the total relative displacement between the platform and the axle boxes to $45 \mathrm{~mm}$. Both bump stops have been modelled by an onedirectional spring that only actuated when the relative displacement was exhausted.

\subsubsection{Buffer gear}

The contact force between two adjacent buffers was simulated by contact-springs. These special springs only generate a resultant contact force when the buffers come into contact with each other. Three-dimensional contact surfaces were used in order to consider the potential contact point between buffers. Friction loss was calculated when the buffers were in contact and, at the same time, slid relative to each other. The model also included the buffers' internal rubber springs that damp the impact when two buffers come into contact. In the simulation models, the buffers were slightly preloaded, so that the buffers were in continuous contact with each other. However, when riding through extremely small curves, the exterior buffers might temporarily loose contact. 


\subsubsection{Coupling screws}

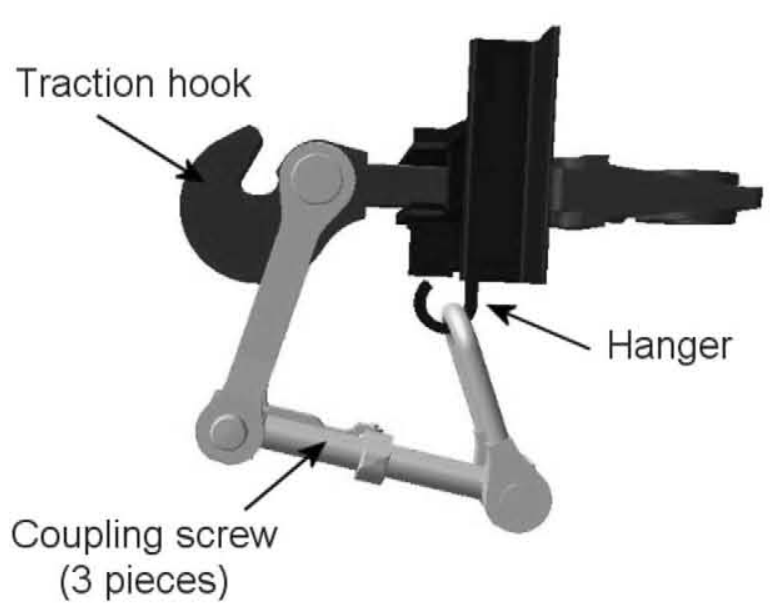

Fig. 3 Components of the un-used coupling screw

The coupling screws were modelled as a three bar linkage whose rigid bars are connected to each other by revolute joints permitting rotation about the lateral axis (see Fig. 3). At the fixation point of the coupling screw to the wagon's traction hook, not only rotation was permitted but also lateral movement representing the clearance that exists there in reality. Friction losses associated to all movements such as rotation and lateral displacement were included in the model.

When a coupling screw is used, its extreme end is placed in the adjacent traction hook (not shown). The coupling screw is then screwed taut until the buffers of two adjacent wagons press into one another, thus preventing oscillation of the wagons at high speeds. In the model, the traction force exerted by the used coupling screw was modelled by a spring that had very high stiffness in all directions.

When the coupling screw is not used, its end is placed in the hanger as shown in Fig. 3. The unused coupling screw additionally incorporated a contact model that simulate the placement in the hanger. The model consisted of four contact volumes that adapted to the curved form of the hanger. Four contact springs placed between the coupling screw's extreme end and the hanger's contact volumes were used in order to calculate the contact between coupling screw and hanger.

\subsubsection{Traction hooks}

The internal rubber spring used to damp the longitudinal forces that arise in the traction hook when two adjacent wagons are coupled to each other, were included in the vehicle model.

\subsubsection{Tank Container}

The tank container was fixed to the freight wagons' platforms allowing longitudinal movement of $40 \mathrm{~mm}$ permitted by the clearance existent in reality between platform and tank container. Bump stops limit this longitudinal motion. Friction loss in this relative movement was also included in the model.

As a next step, an equivalent fluid model, described in section 3, was incorporated in the train vehicle model. Then simulations were run, obtaining first results. However, due to the high number of details included in the multibody model, computation time was high, and simulations were time consuming. Hence, various series of simulations were carried out in order to find out which of the 
details described above could be neglected without altering significantly simulation results. A reduced model was formed.

The following section provides a brief listing of those details that were neglected in the reduced model.

\section{$2.2 \quad$ Reduced model}

After carrying out various comparative simulations, it was found that the presence or negligence of the following details did not alter the vehicle's behaviour that was analysed in this study. They were therefore altered as indicated:

- Buffer gear:

- Coupling screws:

- Used coupling screws:

- Traction hooks:

- Tank containers:
No internal springs

No lateral clearance

The three bars were modelled as one rigid bar

No internal rubber springs

No longitudinal clearance

The reduced, four-wagon-model, had the same number of rigid bodies as the detailed model (124 bodies) but only 79 DOF. Both the reduction of DOF and the omission of force elements led to lower calculation times.

\section{DEVELOPMENT AND INCORPORATION OF THE FLUID MODEL}

Freight trains carrying tank containers are subject to a phenomenon called "sloshing", referring to the oscillation of the liquid contained inside the tank when it is partially filled [5]. The sloshing movements of liquids inside containers are usually simulated by means of highly sophisticated finite element computer programs able to solve the complex fluid flow equations, such as Navier-Stokes equations. Since it is rather difficult to integrate such complex finite element fluid flow models into a multibody system simulation program, an equivalent multibody model was sought that would be able to represent the liquid's sloshing and its interaction with the vehicle [6-11].

In [6] it is shown that only the first eigen frequency of a liquid moving in a tank container is important when studying sloshing phenomena, since the effective modal masses associated to the second and higher order frequencies are negligible in comparison to the first one. Hence, appropriate equivalent models are one-degree-of-freedom models such as a "pendulum" or a "mass-spring" model. In the present study, the "pendulum model" was used to analyse the influence of the liquid's motion on lateral scenarios, such as curve negotiation, and the "mass-spring model" was applied when studying the longitudinal behaviour of the freight trains, such as braking and acceleration manoeuvres.

\subsection{Lateral sloshing model}

A valid model for studying lateral sloshing in tank containers is the so-called "pendulum model". In the present study, the "pendulum model" developed by Ranganathan [7] was used. This model basically consists of a pendulum with a length, $l_{p}$, a point mass, $m_{p}$, attached at its free end, representing the fraction of the liquid that takes part in the oscillating motion, and a point mass, $m_{0}$, fixed to the tank at a certain height, $h_{0}$, representing the stationary part of the fluid. 

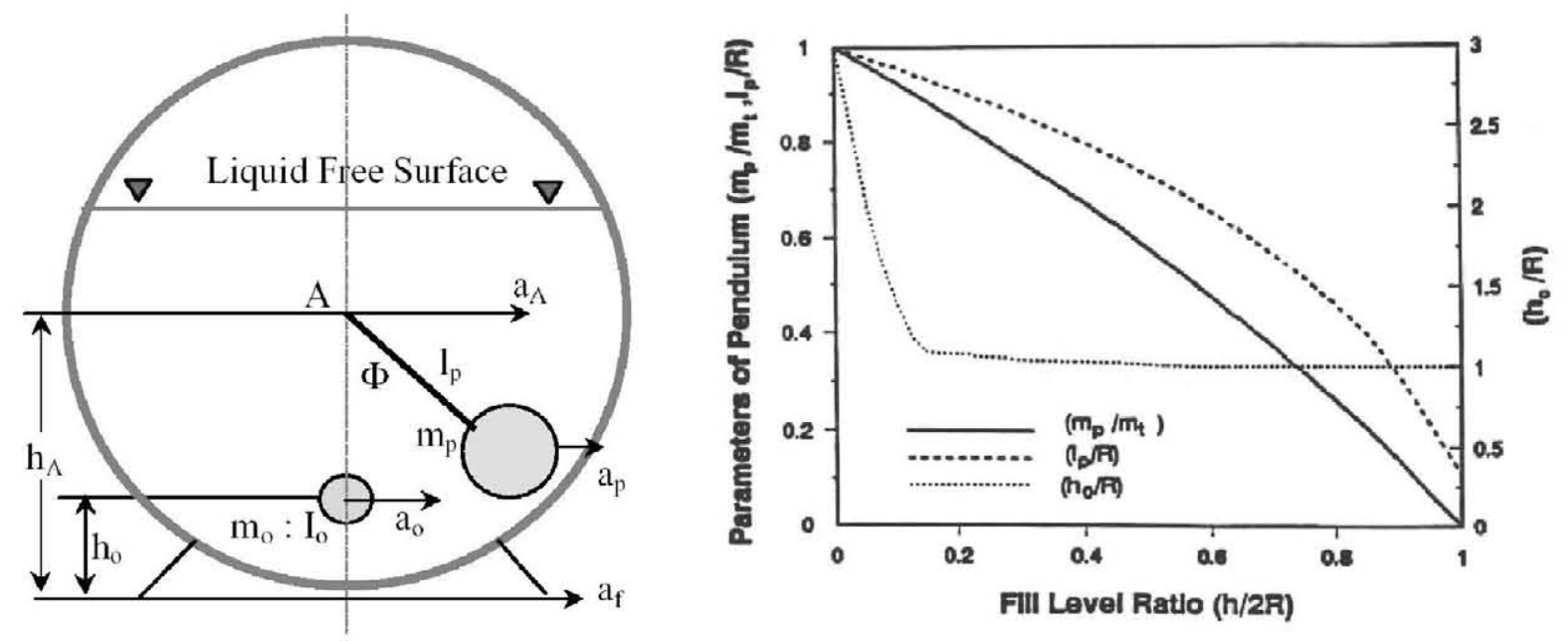

Fig. 4 Pendulum model and parameter chart [7]

Ranganathan provided a chart (Fig. 4) for obtaining values for pendulum length, pendulum mass, etc. in dependency of the container's fill level. An equivalent multibody pendulum model was set up in SIMPACK applying the values given by Ranganathan.

In order to verify the values provided by Ranganathan, a two-dimensional finite element ANSYS model of the partially filled tank container has been established (see Fig. 5).
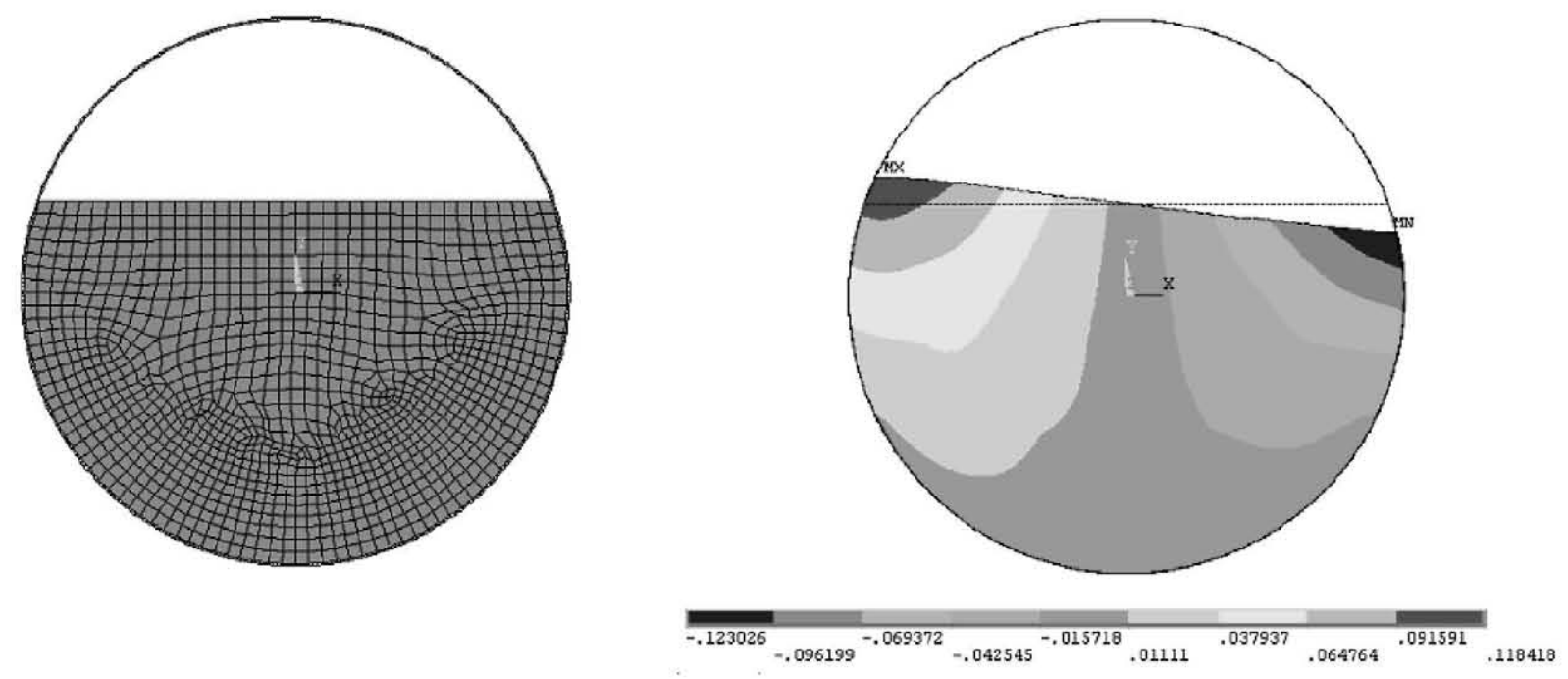

Fig. 5 Two-dimensional ANSYS model of a partially filled tank container, mesh (left) and first mode (right)

Lateral liquid motion was studied in both the Ranganathan pendulum model and the FEM model. The first natural frequency (eigen frequency) obtained in both models was compared, as well as the reaction forces on the tank container walls in lateral and vertical directions. The simulation results showed good agreement. The first eigen frequencies of the SIMPACK pendulum model and of the finite element model were very close to each other and had a relative error of just $2.6 \%$. Comparison of the reaction forces also showed good agreement after reaching the steady state, as can be seen in Fig. 6 and Fig. 7: 


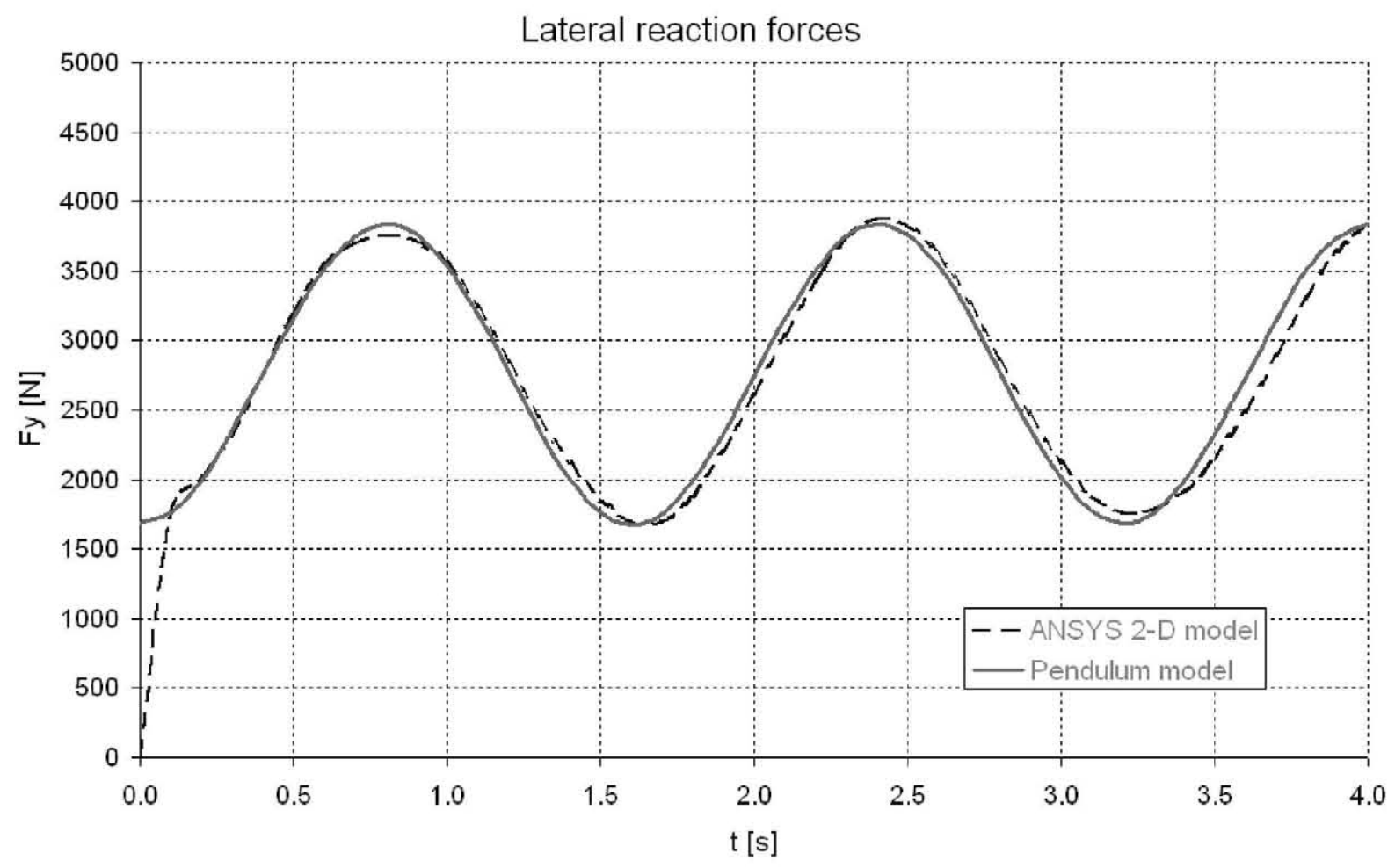

Fig. 6 Comparison of lateral reaction forces

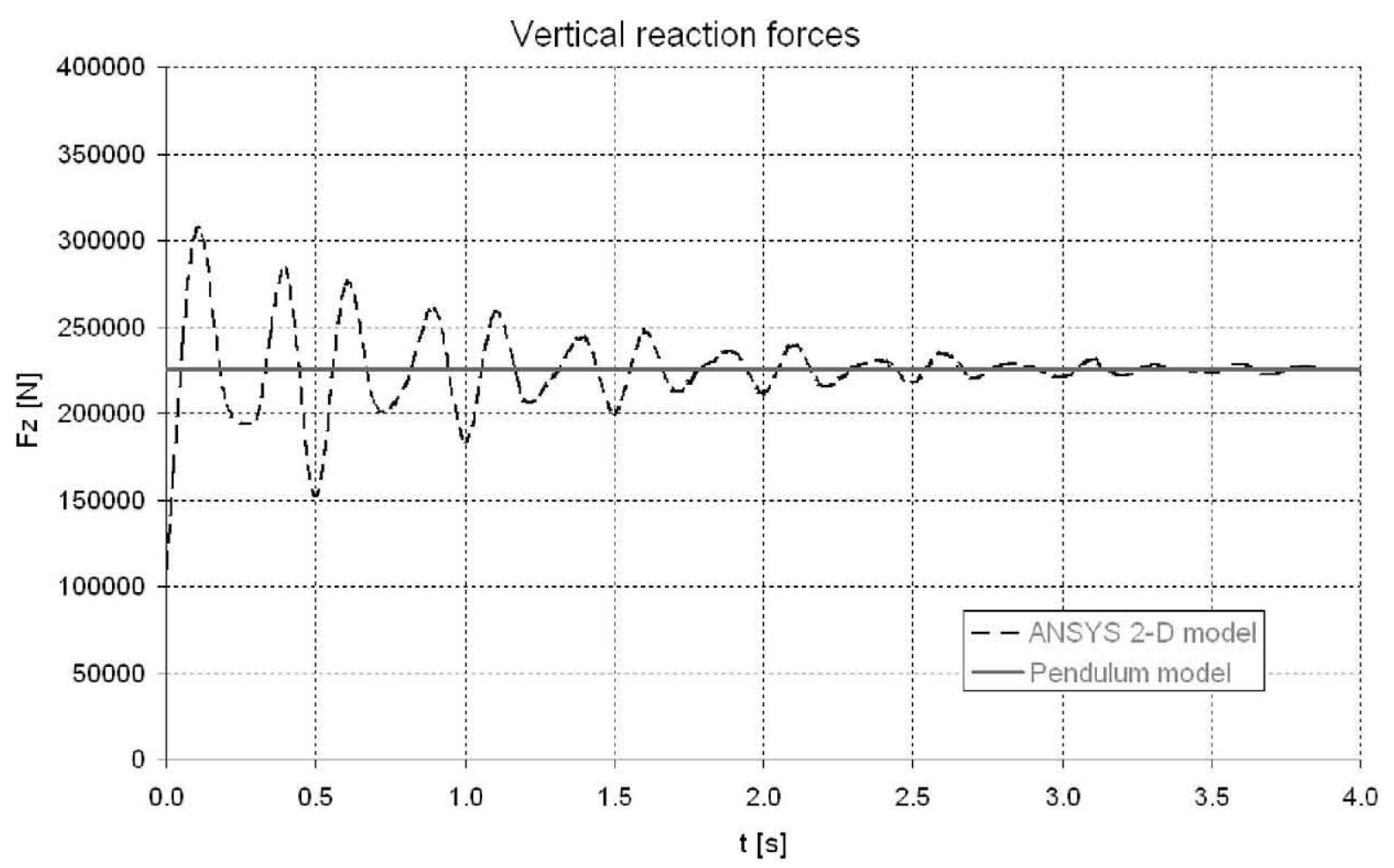

Fig. 7 Comparison of vertical reaction forces

As can be seen in Fig. 7, the vertical reaction force obtained in the FEM-model presents an oscillating motion during the first three seconds. This oscillation can be attributed to the more realistic fluid description in the FEM-model. Although vertical reaction forces are of less importance than transversal ones when studying lateral sloshing, the authors verified that the observed oscillation did 
not affect overall vehicle safety. Some preliminary calculations were performed in which the wheel unload coefficient, $\Delta \mathrm{Q} / \mathrm{Q}$, was analysed. The results have shown that the coefficient always remained below the limit value given by the European standards for approval of railway vehicles [12], $\Delta \mathrm{Q} / \mathrm{Q} \leq 0.6$.

\subsection{Longitudinal sloshing model}

Abramson [6] developed a model that approximates to the motion of a liquid contained in a rectangular cross section tank. Fig. 8 shows the mass that represents the part of the fluid that moves longitudinally, $m_{l}$, and the remaining fluid mass that stays stationary, $m_{0}$.

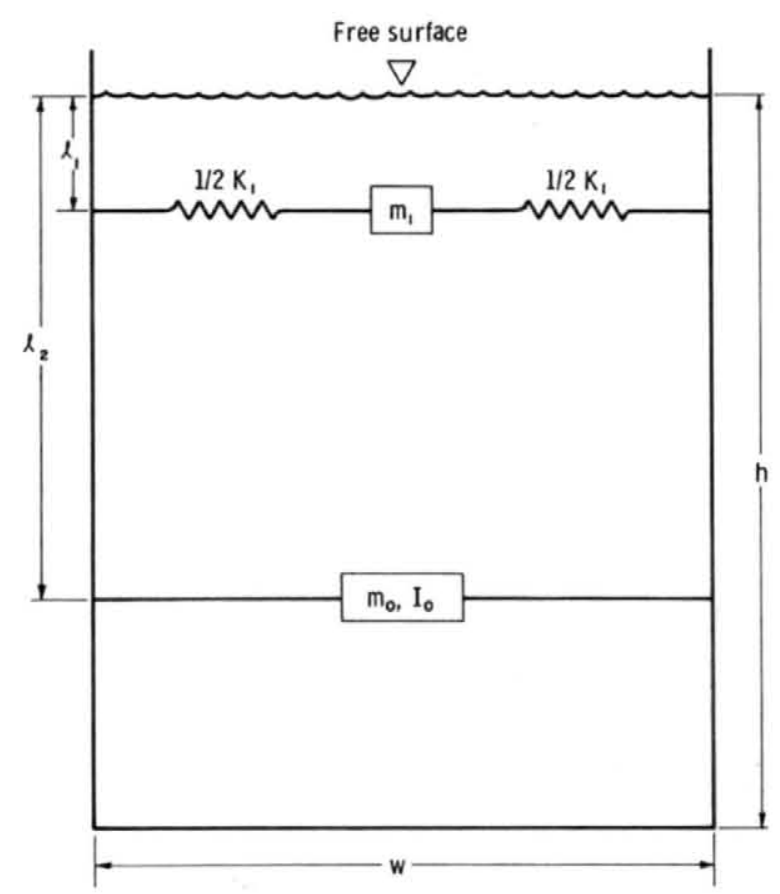

Fig. 8 Mass-spring model [6]

Abramson [6] proposed a set of equations that allow the calculation of values for the equivalent masses, their corresponding positions, and the spring stiffness:

$$
\begin{aligned}
& m_{t}=\rho \cdot h \cdot w \cdot b \\
& m_{1}=m_{t} \cdot\left(\frac{w}{3,87 \cdot h}\right) \cdot \tanh \left(3,14 \cdot \frac{h}{w}\right) \\
& m_{0}=m_{t}-m_{1} \\
& k_{1}=m_{t} \cdot\left(\frac{g}{1,23 \cdot h}\right) \cdot\left(\tanh \left(3,14 \cdot \frac{h}{w}\right)\right)^{2} \\
& l_{1}=\frac{w}{1,57} \cdot \tanh \left(1,57 \cdot \frac{h}{w}\right) \\
& l_{2}=\frac{h}{2}+\frac{m_{1}}{m_{o}} \cdot\left(\frac{h}{2}-l_{1}\right)
\end{aligned}
$$

Where: 
- $\quad m_{t}$ is the total liquid mass

- $\rho$ is the liquid's density

- $\quad h$ is the height of the liquid's free surface

- $w$ is the length of the rectangular tank

- $\quad b$ is the width of the rectangular tank (not shown in Fig. 8)

- $g$ is the gravitational acceleration

And where the unknowns are:

- $\quad m_{1}$, oscillating mass

- $\quad m_{0}$, fixed mass

- $k_{1}$, total spring stiffness

- $l_{2}$, distance of the fixed mass from the liquid's free surface

- $l_{l}$, distance of the oscillating mass from the liquid's free surface

The equations' solution depends on the container's rectangular cross section and overall length, as well as on the liquid's density and the fill level. Given that the tank containers analysed in the present paper were of circular cross section, a study was carried out in order to find out which rectangular cross section assimilated best to the circular cross section, both qualitatively and quantitatively. Since the longitudinal liquid motion inside the tank was now of interest, a two-dimensional approach was no longer valid, and three-dimensional finite element models of the original circular tank container and of equivalent rectangular ones were set up in ANSYS and results were compared.
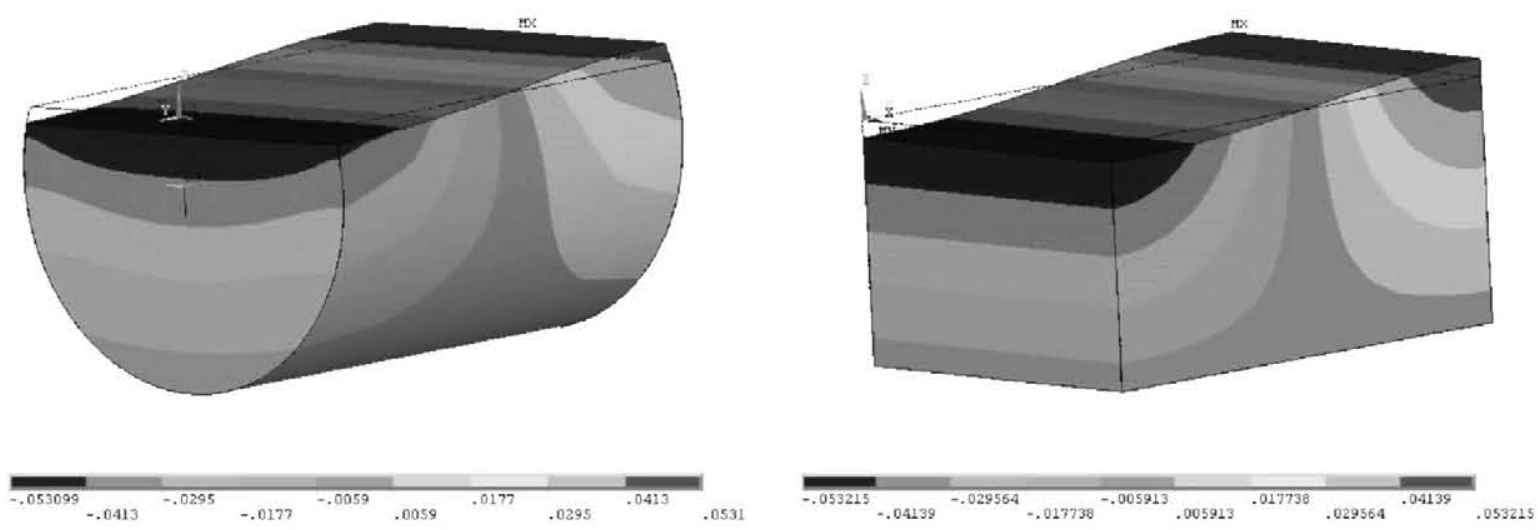

Fig. 9 Three-dimensional finite element ANSYS models

Several rectangular sections of different dimensions were analysed. It was shown that the rectangular section that provides most accurate results is the one that lies between the rectangles that inscribe and circumscribe the circular cross section. In Fig. 10, the original circular section is represented by a dotted line and the mid-rectangular shape by a bold (blue) line. 

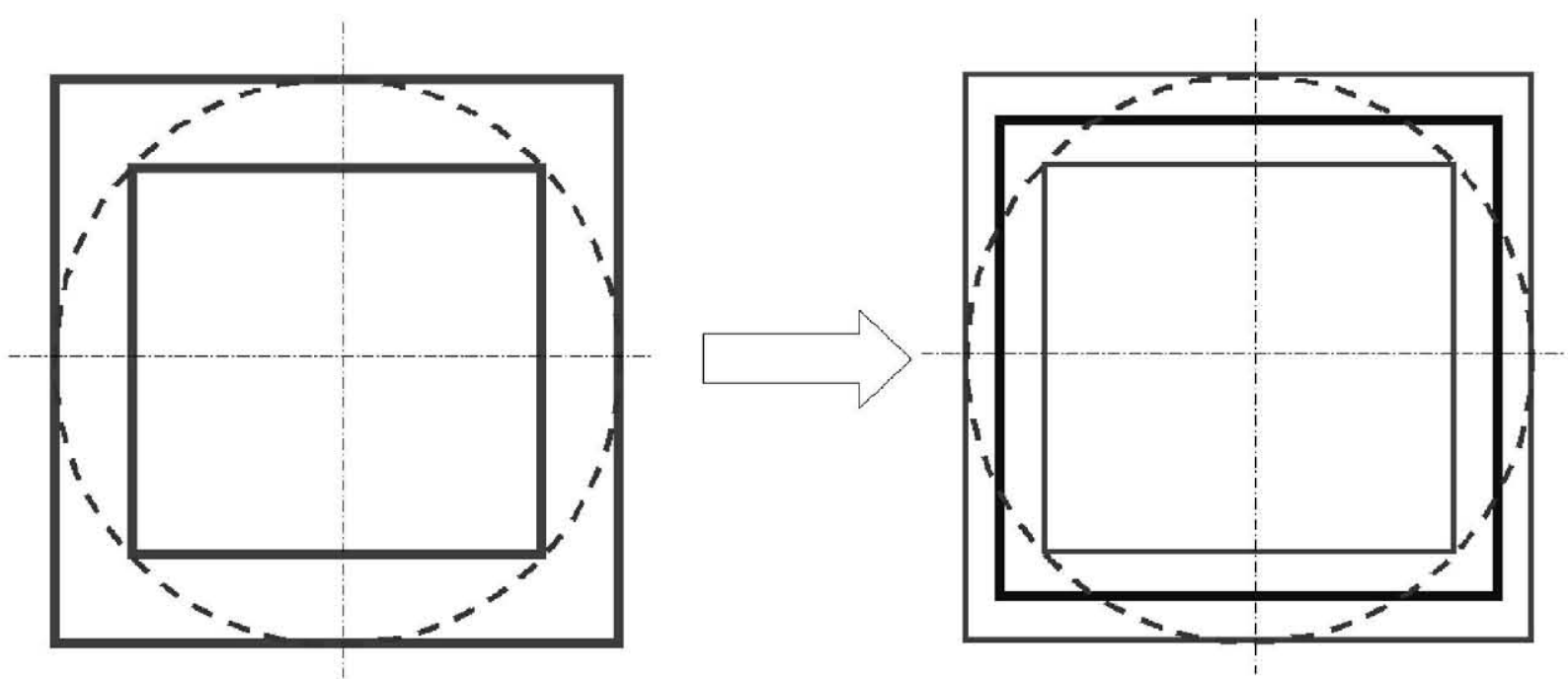

Fig. 10 Inscribed, circumscribed and circular cross sections (left), mid-rectangular cross section (right, bold)

The circular and the mid-rectangular finite element models were compared with respect to the first longitudinal sloshing eigen frequency, as well as to longitudinal reaction forces, when applying a sinusoidal longitudinal acceleration to the tanks, calculated at the tanks' domes. The longitudinal forces at the tanks' domes were very similar as shows Fig. 11.

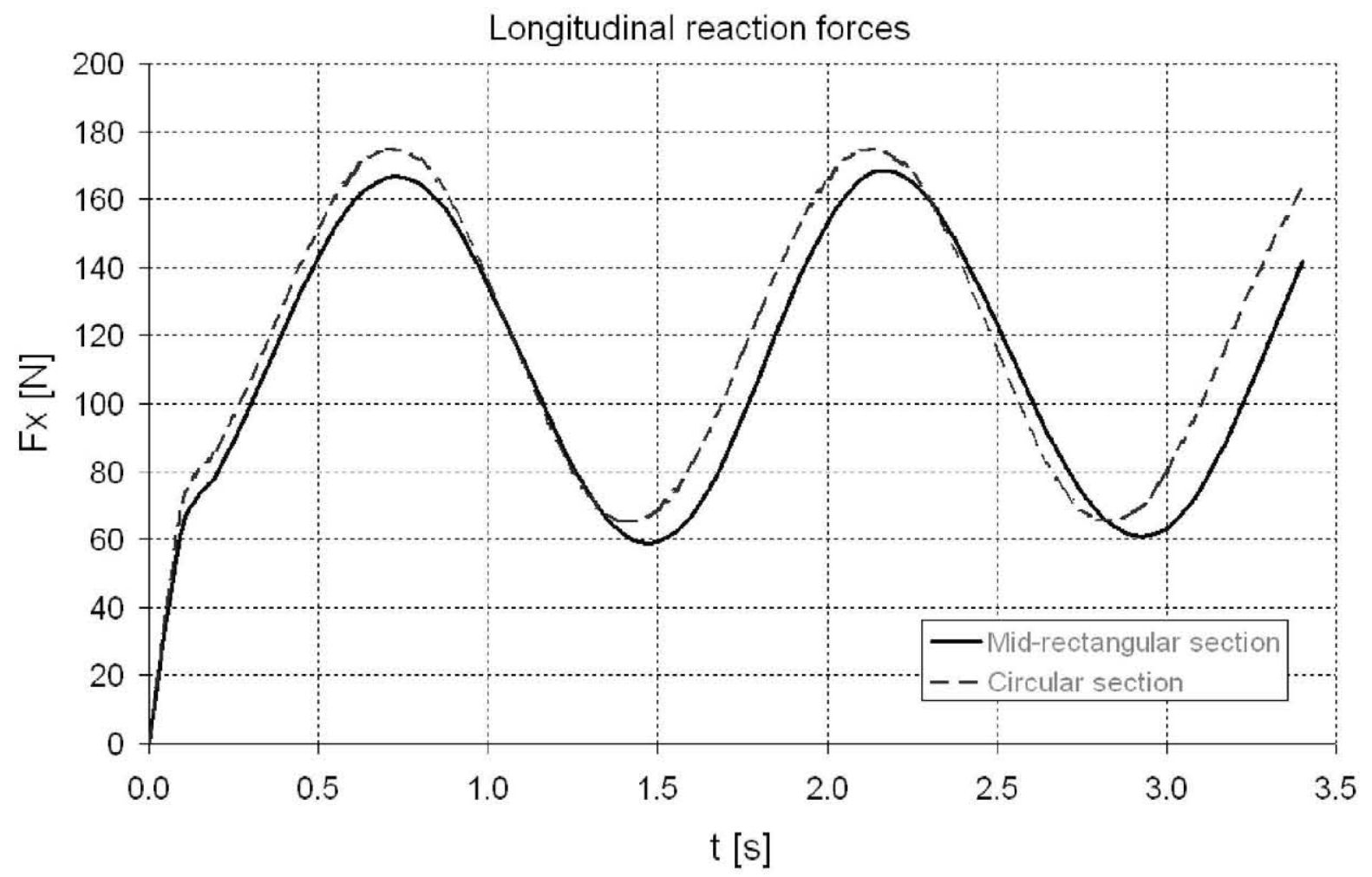

Fig. 11 Longitudinal reaction force

The first longitudinal eigen frequency also showed very good agreement between the circular and the mid-rectangular cross sections. For this reason, the mid-rectangular section was used when calculating the equivalent model's parameters given by Abramson [6]. The equations proposed by [6] were then resolved and the following results were obtained: 


$$
\begin{aligned}
& m_{t}=25056 \mathrm{~kg} \\
& m_{1}=6847 \mathrm{~kg} \\
& m_{0}=18209 \mathrm{~kg} \\
& k_{1}=111160 \mathrm{~N} / \mathrm{m} \\
& l_{1}=1.053 \mathrm{~m} \\
& l_{0}=0.721 \mathrm{~m}
\end{aligned}
$$

These values were used in the mechanical equivalent model that was incorporated in the MBSvehicle model in order to analyse the vehicle's longitudinal behaviour. In the simulations that were performed, the liquid oscillated longitudinally in the tank container with a first eigen frequency of $0.64 \mathrm{~Hz}$, as was predicted by the equation for the free oscillation of a simple mass:

$$
f=\frac{1}{2 \cdot \pi} \sqrt{\frac{k_{1}}{m_{1}}}=0.64 \mathrm{~Hz}
$$

Other approaches for analytical calculation of equivalent models that represent longitudinal fluid motion in horizontal circular cross section tanks can be found in $[7,8]$.

\subsubsection{Tank containers with vertical, equally spaced baffles}
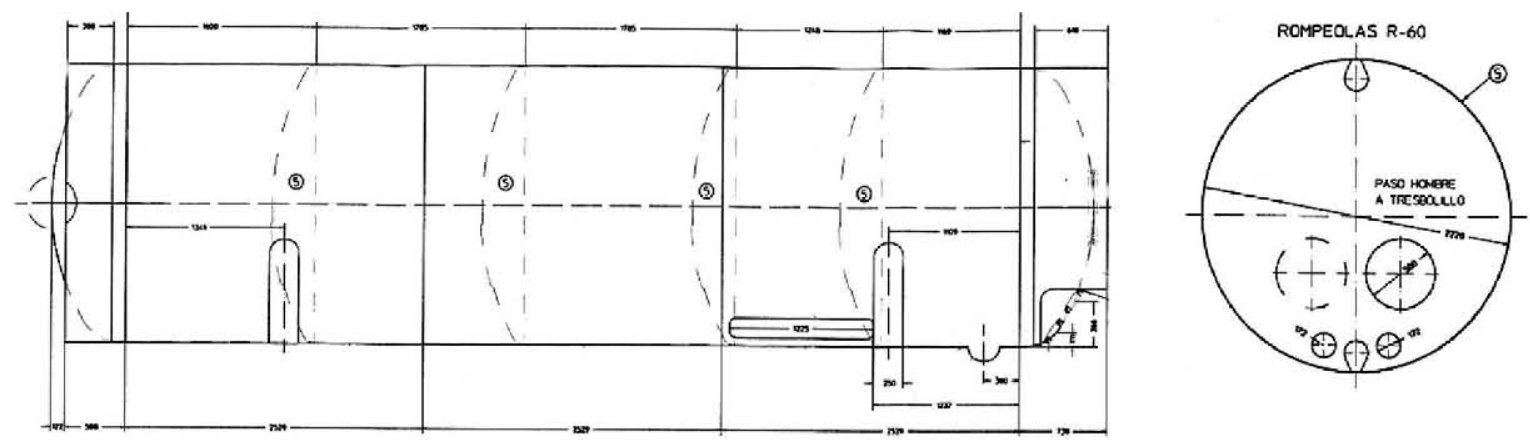

Fig. 12 Tank container with vertical baffles

In a next step, the influence of baffles on longitudinal liquid sloshing was analysed. The tank containers were equipped with four vertical, equally spaced baffles that presented a hole opening in the lower part, as can be seen in Fig. 12. These openings allow the liquid to flow from one compartment into another. However, a finite element fluid flow analysis showed that the fluid exchange through the holes was negligible. Therefore, the baffles were considered as solid walls, dividing the container into five separate compartments, each filled with a fifth part of the entire liquid load. In the multibody model, an equivalent mass-spring model was incorporated for every one of the five compartments, as can be seen in Fig. 13. 

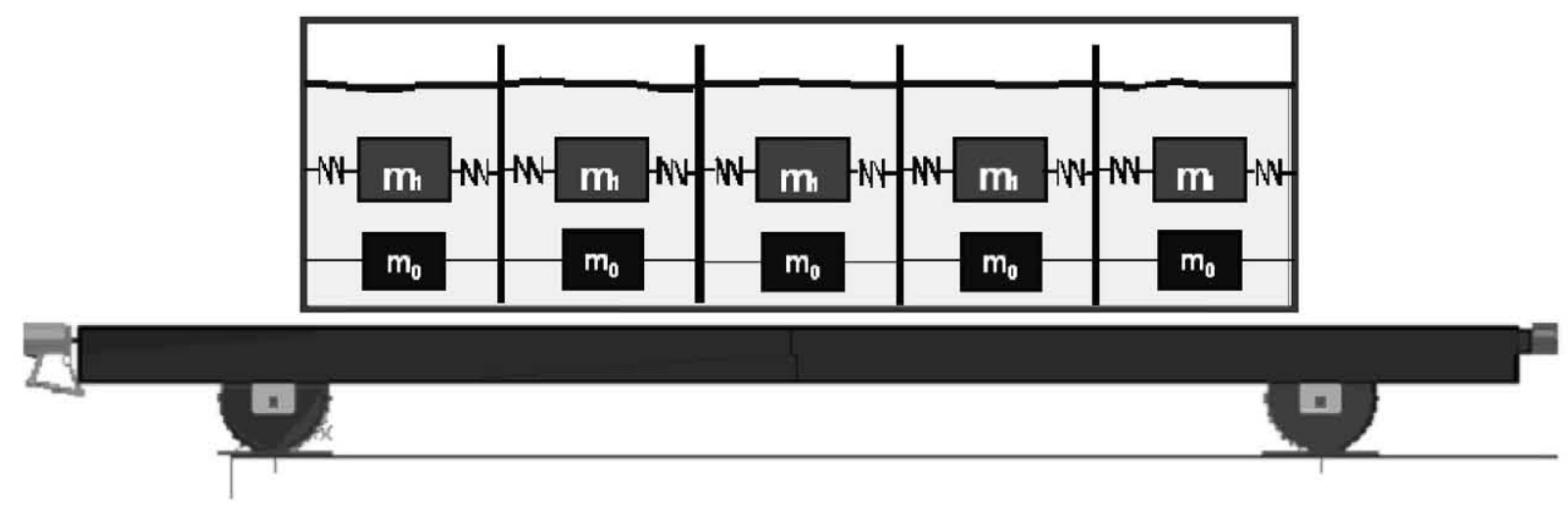

Fig. 13 Multi-body system model of a container freight wagon with five compartments

Solving the Abramson-equations in the same manner as for the container without baffles, described in section 3.2, gave the following results for each one of the compartments:

$$
\begin{array}{ll}
m_{t, \text { one compartmen }}=5011.2 \mathrm{~kg} & =m_{t} / 5 \\
m_{1, \text { one compartmen }}=1369.4 \mathrm{~kg} & =m_{1} / 5 \\
m_{0, \text { onecompartmen }}=3641.8 \mathrm{~kg} & =m_{0} / 5 \\
k_{1, \text { one compartmen }}=22232 \mathrm{~N} / \mathrm{m} & =k_{1} / 5 \\
l_{1, \text { onecompartmen }}=1.053 \mathrm{~m} & =l_{1} \\
l_{0, \text { one compartmen }}=0.721 \mathrm{~m} & =l_{0}
\end{array}
$$

It can be seen that the fraction of the liquid moving within each of the five compartments, oscillated at the exact same frequency as did the entire liquid oscillating in the tank without baffles:

$$
f_{\text {baffles }}=\frac{1}{2 \cdot \pi} \sqrt{\frac{k_{1, \text { one compartmen }}}{m_{1, \text { one compartmen }}}}=\frac{1}{2 \cdot \pi} \sqrt{\frac{k_{1} / 5}{m_{1} / 5}}=0.64 \mathrm{~Hz}
$$

Hence, the vertical baffles do not alter the first longitudinal eigen frequency of the liquid motion. However, they lower the impact that the moving liquid contained in each compartment exerts on the domes and the baffle walls.

\section{DYNAMIC BEHAVIOR SIMULATION}

The final SIMPACK model consisted of tank container wagons that were filled with a liquid load up to $87 \%$. The reduced vehicle model, as described in section 2.2 , was used for analysis. 


\subsection{Curve negotiation}

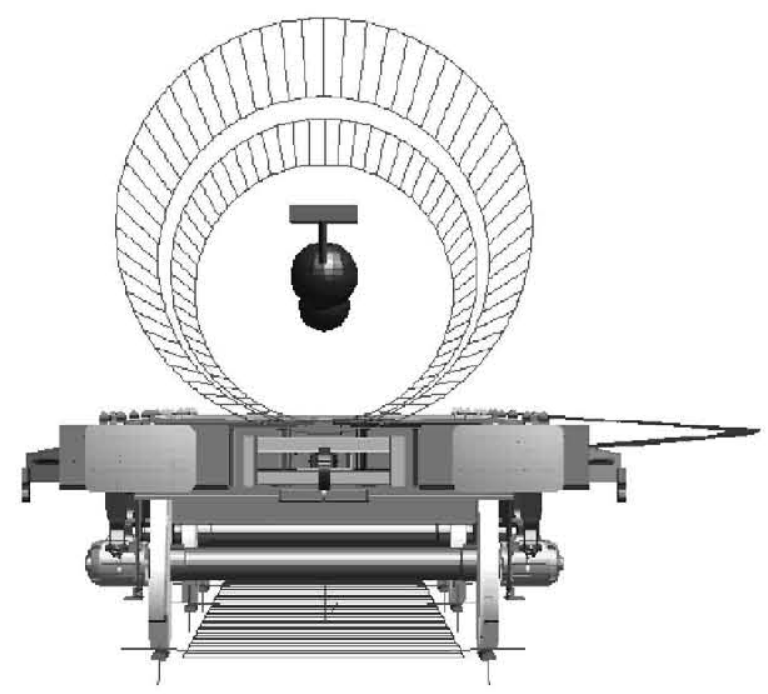

Fig. 14 Two wagon SIMPACK model for analysis in curves including pendulum

The "pendulum model", described in section 3.1, was incorporated in the SIMPACK train model (Fig. 14) when running through curves, since the centripetal acceleration experienced in curves provokes a lateral oscillatory motion of the containers' liquid load. Various simulations were carried out in order to study the influence of the lateral liquid sloshing on the dynamic train behaviour. The following worst-case scenario was chosen in simulation: Passing through an " $\mathrm{S}$ "-shaped curve of radii $R=500 \mathrm{~m}$ at the maximum train velocity of $v=120 \mathrm{~km} / \mathrm{h}$.

Results have shown that the liquid's lateral sloshing motion did not influence notably on overall train behaviour, the freight train composition passed through the curve without showing either high lateral accelerations or dangerous wheel unloading due to liquid load sloshing, thus lateral train safety was not affected.

\subsection{Braking and acceleration maneuvers}

When studying the influence of braking and acceleration manoeuvres on straight track, the longitudinal "mass-spring model" (described in section 3.2) was incorporated in the SIMPACK freight train model, since only longitudinal dynamics were affected.

When the four wagon train composition was subject to braking and acceleration manoeuvres on a straight track, not only the train's velocity changed but also the liquid contained in the tanks was set into longitudinal oscillatory motion. This longitudinal sloshing motion caused a shifting of the centre of gravity of the liquid load from the tank's centre toward one of its domes. Occasionally, this phenomenon can lead to undesirable unloading of wheelsets. However, it was observed in this analysis that train safety was not affected by wheelset unloading caused by longitudinal liquid sloshing. It was also noted that under particular conditions, the un-used coupling screw began a considerable oscillatory motion. In severe cases this oscillation could lead to the coupling screw's undesirable release from its hanger, followed by a free pendulum-like motion of the coupling screw around its attachment point (see Fig. 15) possibly hitting nearby components. 


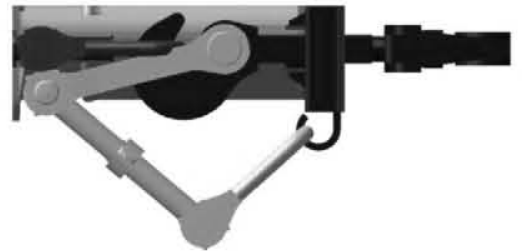

$\mathrm{t}=0.0 \mathrm{~s}$

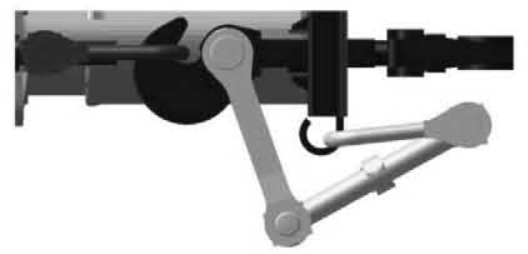

$\mathrm{t}=0.6 \mathrm{~s}$

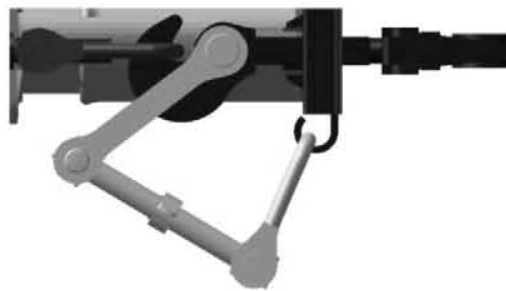

$\mathrm{t}=0.2 \mathrm{~s}$

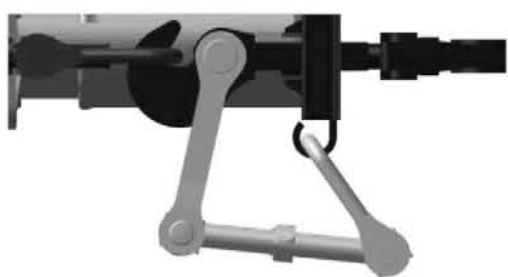

$\mathrm{t}=0.9 \mathrm{~s}$

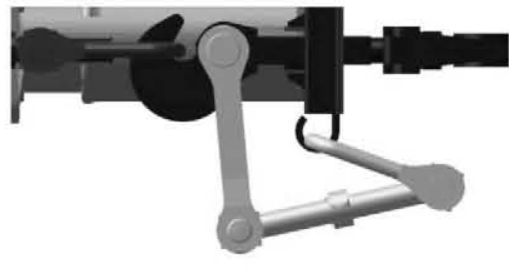

$t=0.5 \mathrm{~s}$

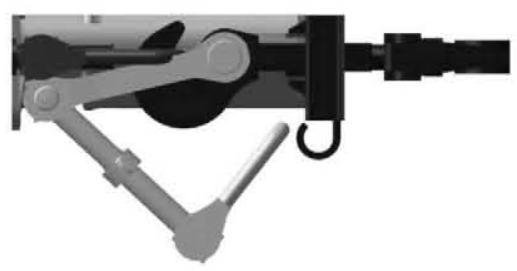

$t=1.2 \mathrm{~s}$

Fig. 15 Swinging motion of the coupling screw

\subsubsection{Factors favouring the coupling screw's release}

Since the once released, un-used coupling screw could possibly hit nearby components, the problem was further analysed. It was noted that the tendency of the coupling screw to jump out of its hanger increased with higher values for acceleration and braking (up to $1 \mathrm{~m} / \mathrm{s}^{2}$ ), and also depended on the sequence of acceleration and braking; when intense braking followed a period of acceleration, the coupling screw was more likely to jump out of its hanger due to high amplitudes in the coupling screw's swinging motion. The train's velocity also influenced the coupling screw's behaviour; at higher velocities, more important amplitudes of the coupling screw's oscillatory motion were reached more easily.

\subsubsection{Influence of the liquid load}

The above-mentioned phenomenon of the coupling screw's swinging motion with high amplitudes was only observed in simulations when a particular type of liquid was used in combination with containers of certain dimensions and filling heights. For example, high oscillations were obtained for liquids with a density close to the density of water $\left(1000 \mathrm{~kg} / \mathrm{m}^{3}\right)$ and for filling levels of about $85 \%$ in combination with the containers shown in Fig. 1. When the cisterns were filled with liquids of different characteristics or when the filling level or the tank dimensions were altered, the coupling screw's rotation did not reach the observed high amplitudes, and thus it did not jump out of its hanger. Therefore, it was analysed to what degree the longitudinal sloshing motion of the liquid inside the containers was the reason for the coupling screw's high oscillation.

When repeating simulations without liquid load, the coupling screw's motion was less severe and it ceased to jump out of its hanger. The same observation was made when the liquid load was replace by a solid load. Therefore, it was reasoned that the longitudinal liquid sloshing was the origin for the coupling screw's critical oscillatory motion.

It was now studied whether resonance was a factor in the problem. Performing a modal analysis, the first longitudinal eigen frequency of the liquid was found to be $f_{\text {liquid }} \approx 0.64 \mathrm{~Hz}$, as had been 
calculated priorly with the Abramson-equations in section 3.2. In order to find the frequency at which the un-used coupling screw oscillated, a model was set up where the liquid load was replaced by an equally heavy solid load, thus suppressing the longitudinal fluid sloshing, while leaving all the other parameters unchanged. The frequency at which the coupling screw oscillated was close to $f_{\text {screw }} \approx 0.7 \mathrm{~Hz}$, almost coinciding with the liquid's first natural frequency. Hence, resonance could be an important factor in the problem.

The following figures show the frequency response of the longitudinal motion of the liquid contained within the tanks (Fig. 16) and the frequencies of the rotational motion of the un-used coupling screw about its hinge (Fig. 17). The presence of baffles does not alter the frequency at which the liquid oscillates.

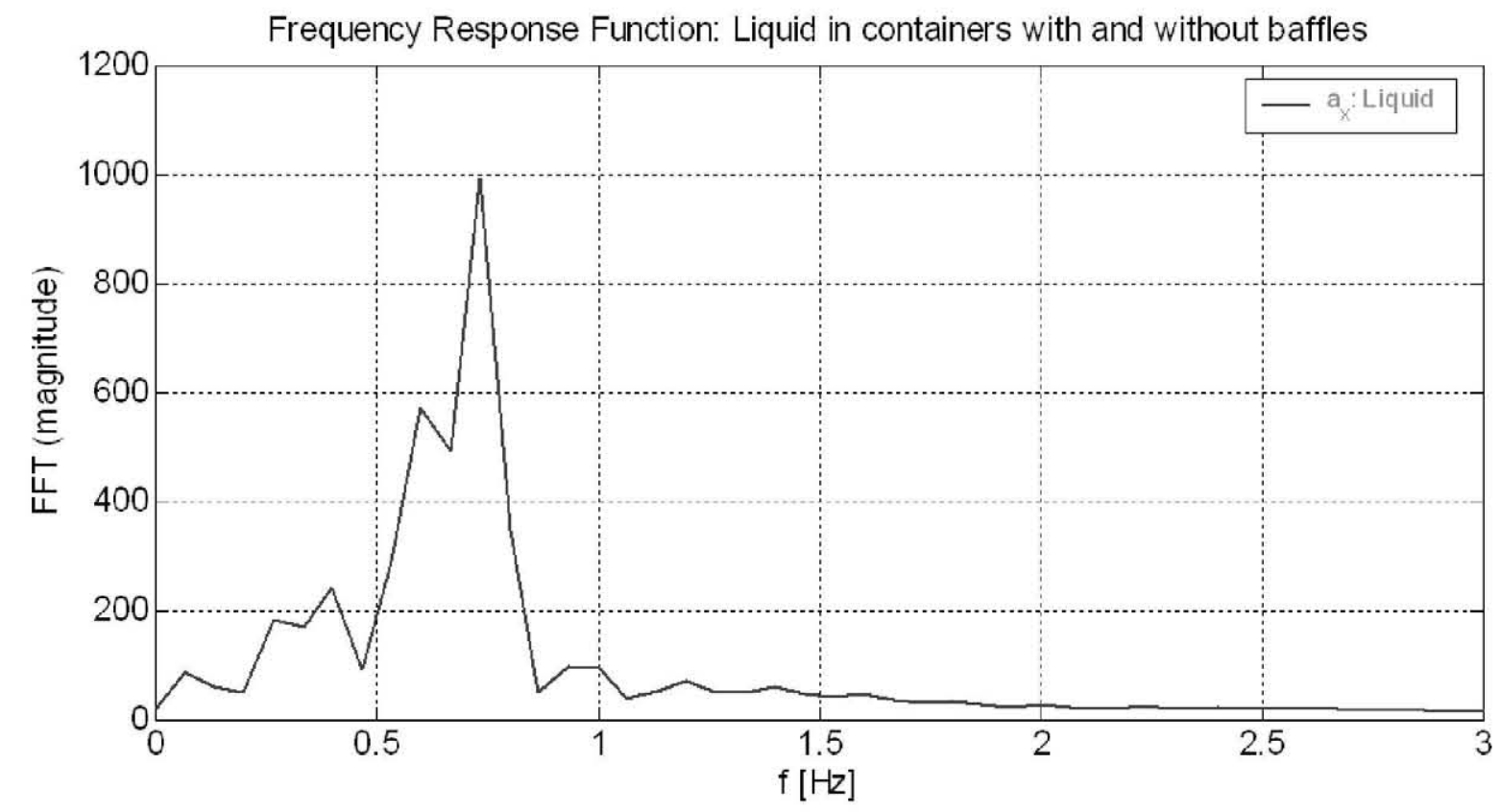

Fig. 16 Frequency response function of the liquid in containers with and without baffles 


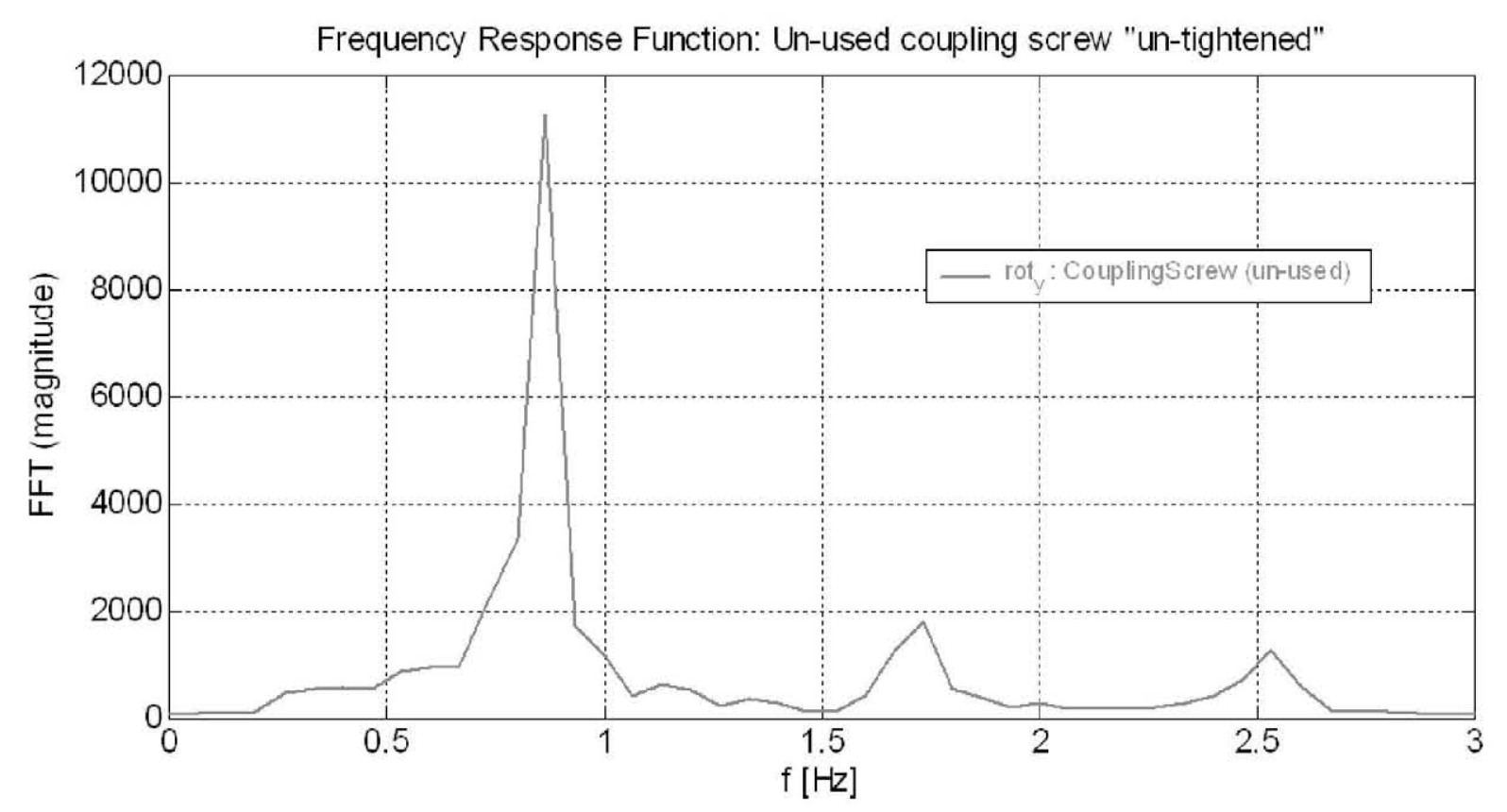

Fig. 17 Frequency response function of the un-used coupling screw in its "un-tightened" position

It can be seen that the first frequency in both cases peaks between 0.6 and $0.8 \mathrm{~Hz}$. When the liquid's first eigen frequency was altered in the simulation models, e.g. by changing the tank's dimensions or the liquid's properties, the coupling screw ceased to jump out of its hanger. Therefore, the authors believe that this unexpected phenomenon could be attributed to a resonance effect originated by a particular configuration of tank dimensions and liquid properties.

\subsubsection{Possible solutions}

In the above-described combination of tank container and liquid load, a resonance effect was observed, leading to the release of some of the coupling screws from their hangers. One way to avoid this undesired although not hazardous release is to change the fluid's first eigen frequency, e.g. by an appropriate calculation of the tanks' dimensions or by implementing properly designed baffles. It was shown in section 3.2.1 that vertical equally spaced baffles, habitually employed in tank containers, did not alter the liquid's longitudinal natural frequencies, and thus did not solve the problematic situation. It could be shown in a separate multibody model, which included tank containers equipped with the described baffles, that the un-used coupling screw also jumped out of the hanger with vertical baffles present.

Another, simpler way is to change the coupling screws configuration in such a manner, that the swinging-like motion is suppressed. Fig. 18 shows a "tightened" un-used coupling screw, which avoids this critical oscillation. 

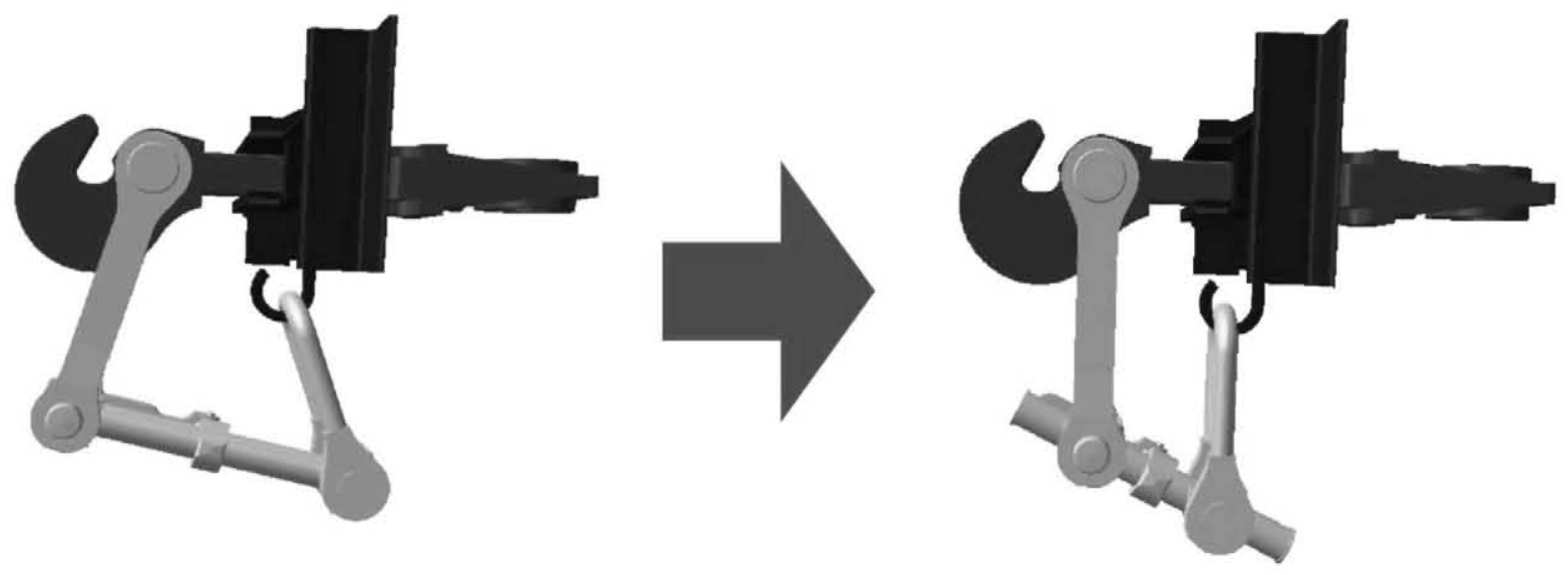

Fig. 18 "Un-tightened" (left) and "tightened" (right) un-used coupling screw

The coupling screw is tightened by fastening the nut located at the centre of the lower bar. When coupling freight wagons, the un-used coupling screw should be "closed" until reaching the tightened position as shown in Fig. 18. However, sometimes it occurs that coupling screws are not completely tightened in the coupling process. Fig. 19 and Fig. 20 compare the contact forces that appear between the coupling screw and its fixation in the hanger for the two configurations mentioned during extreme acceleration and braking manoeuvres.

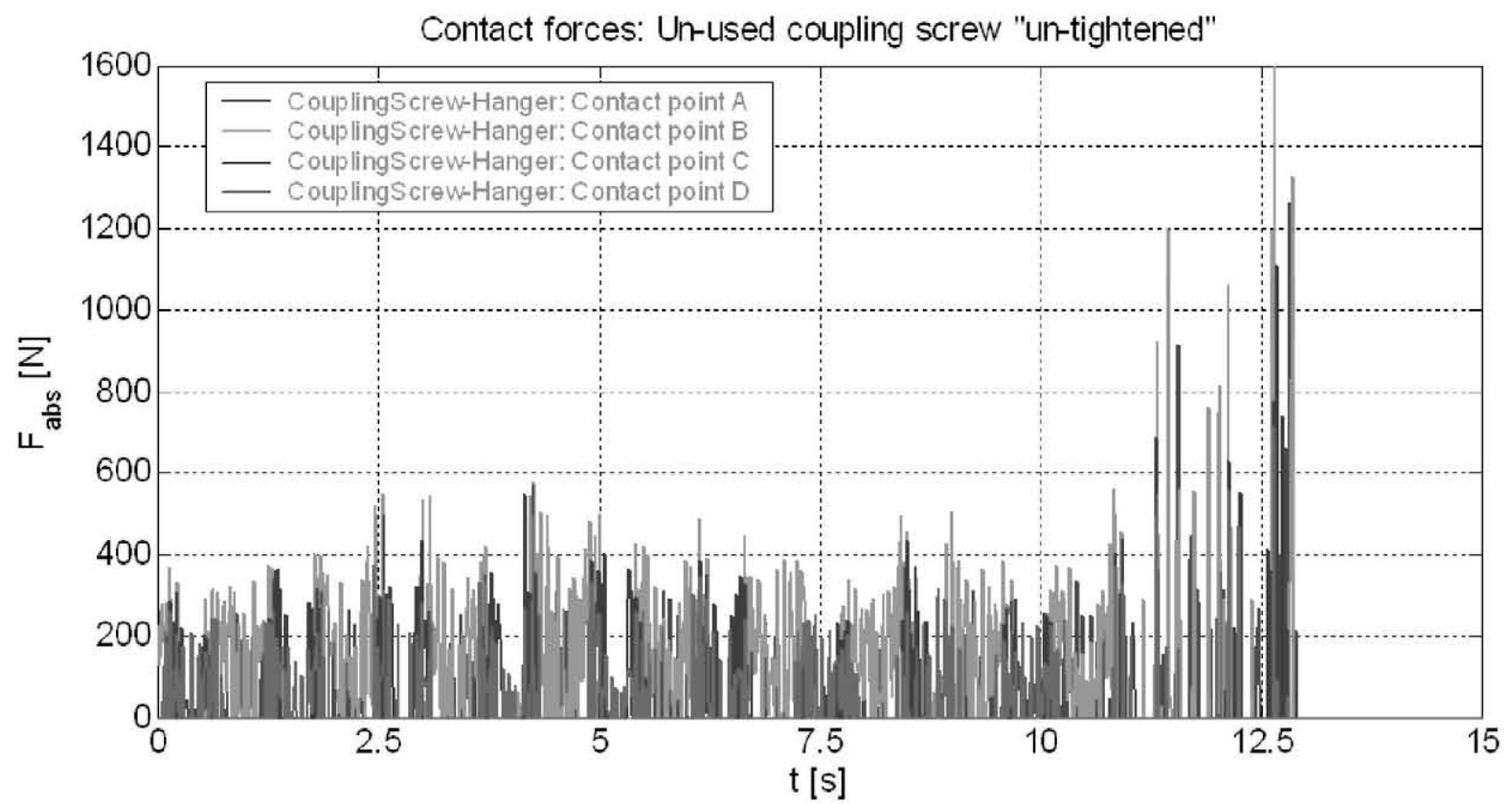

Fig. 19 Contact forces between the un-used coupling screw and its hanger in the "un-tightened" position 


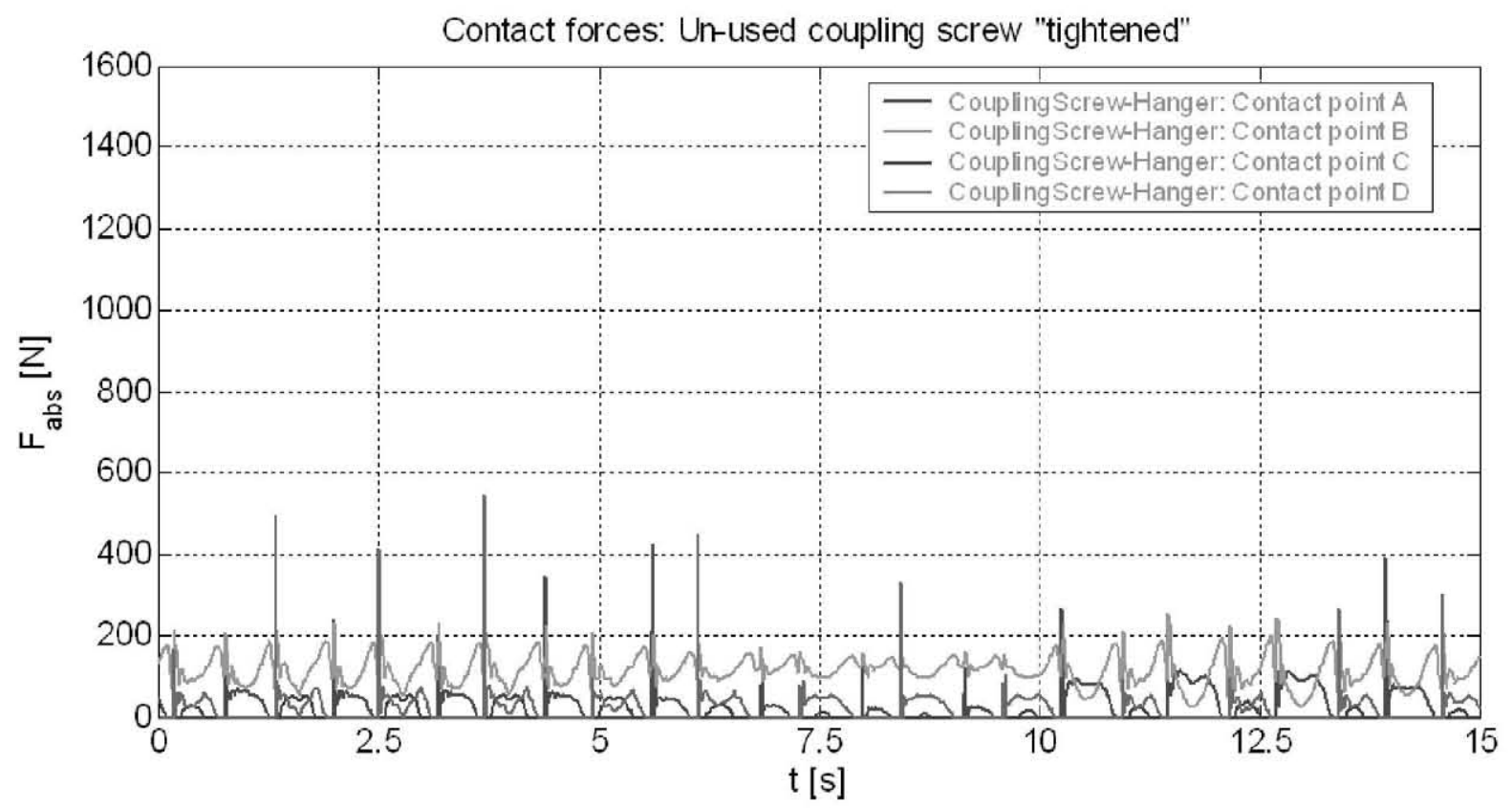

Fig. 20 Contact forces between the un-used coupling screw and its hanger in the "tightened" position

It can be observed that the contact forces were considerably lower in the case of the "tightened" coupling screw. This was due to a less violent oscillation of the coupling screw during the train's acceleration and braking manoeuvres resulting in lower impacts of the coupling screw on the hanger. In case of the "un-tightened" coupling screw, it can be seen in Fig. 19 that at $t \approx 12.8 \mathrm{~s}$ the coupling screw jumped out of its hanger, the contact forces turning to zero.

Fig. 21 and Fig. 22 show the vertical acceleration measured at a point on the hanger, which is located on the extreme end of the wagon's platform for both of the configurations of the coupling screw mentioned above.

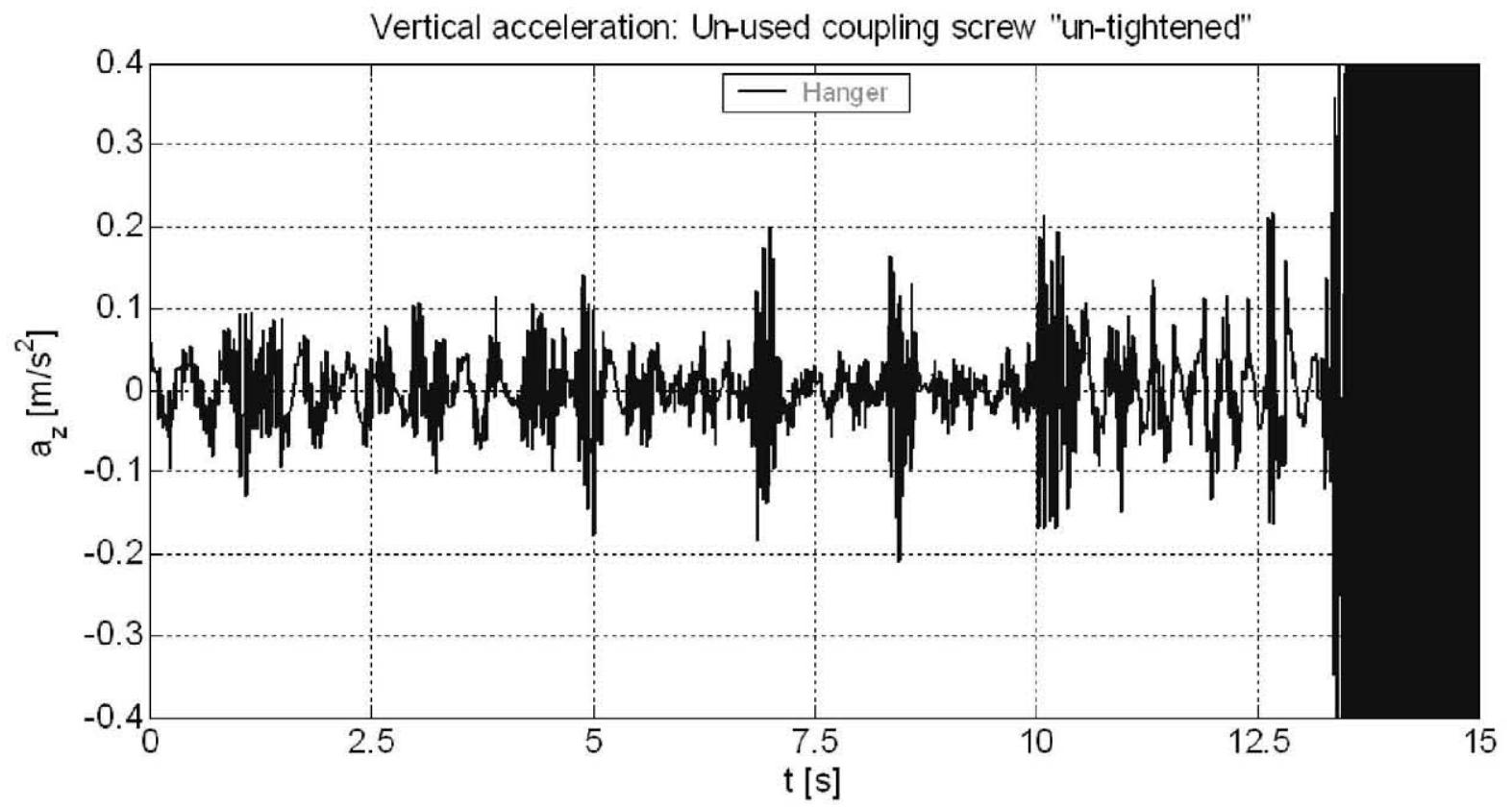

Fig. 21 Vertical acceleration of the hanger measured when the coupling screws are 'un-tightened' 


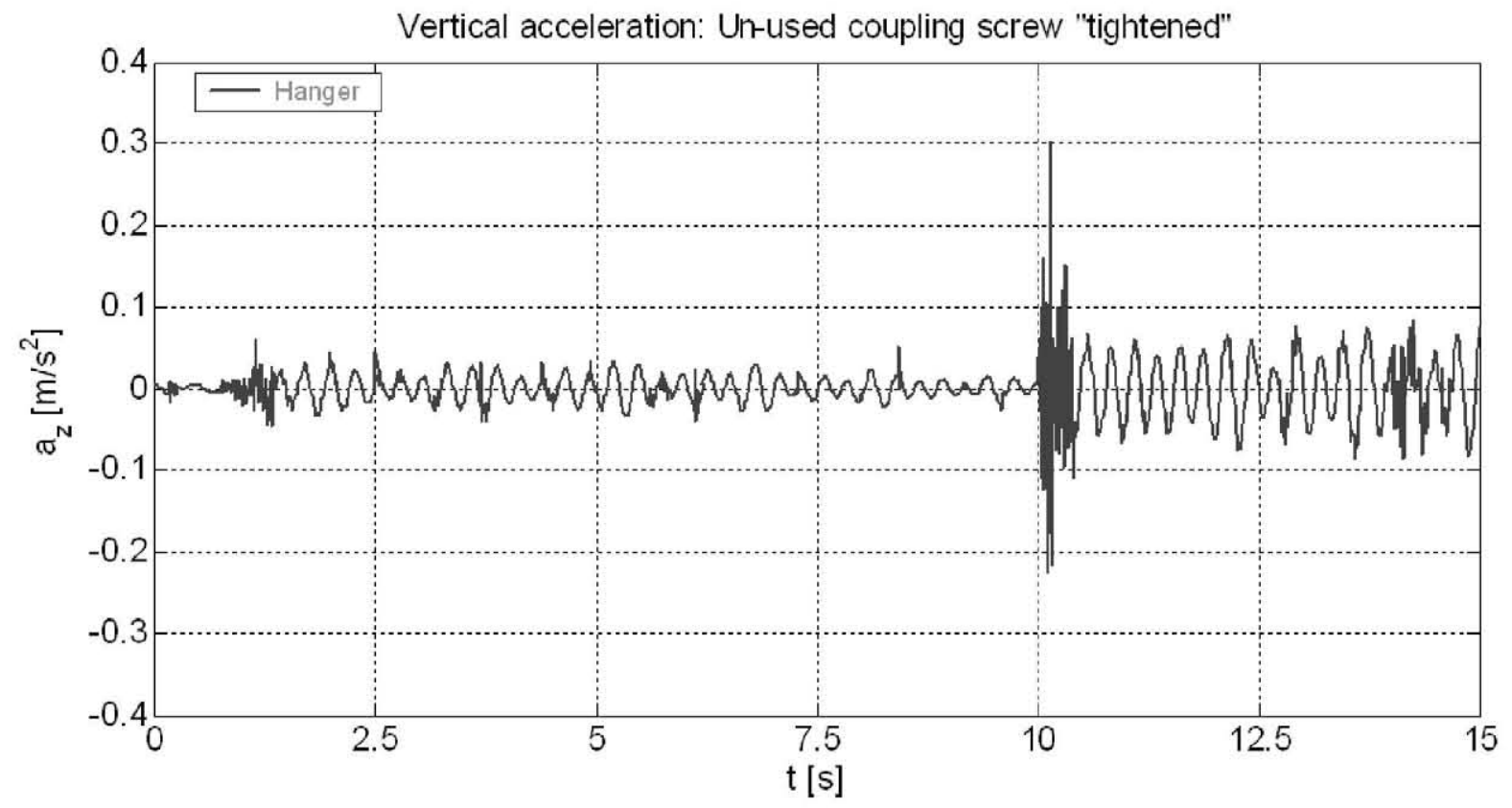

Fig. 22 Vertical acceleration of the hanger measured when the coupling screws are 'tightened'

In the case of the "un-tightened" coupling screw, much higher accelerations can be observed due to the high impacts that the coupling screw exerts on its hanger.

\section{CONCLUSIONS}

Compositions of two-axle freight wagons, carrying partially filled liquid cargo tank containers, were modelled in the multibody program SIMPACK. The liquid contained in the tanks began an oscillatory motion, called "sloshing", when the train experienced external acceleration, such as curve passing or acceleration and braking manoeuvres. It was shown that the sloshing inside the containers did not put in danger the freight train's running safety. However, it was observed that the un-used coupling screws got into resonance with the first eigen frequency of the liquid load's longitudinal motion, leading to the undesired, although not hazardous, release of some coupling screws from their hangers. Solutions were suggested in order to avoid the resonance problem. It should be noted that the resonance observed only occurs in freight trains of a certain combination of factors, such as train and container dimensions, the type of liquid set into motion, the braking and acceleration patterns, etc.

Future research will include a more sophisticated leaf spring suspension model including elastic deformation and friction loss between leaves, as well as the study of a proper baffle design for this kind of railway application.

\section{ACKNOWLEDGMENTS}

The authors gratefully thank the Spanish Railway Operator (RENFE Operadora) for their cooperation and support throughout this project. Particular thanks go to Mr. Antonio Albadalejo (RENFE Operadora, UN Mercancías), Mr. Vicente Cano and Mr. Marco Rodríguez (both RENFE Operadora, UN Mantenimiento Integral de Trenes). Thanks also to Oscar García, for the contributions made to this project as a result of his master thesis. 


\section{REFERENCES}

1 Vera, C., Suárez, B., Paulin, J. and Rodríguez, P. Simulation Model for the Dynamic Study of Overhead Rail Current Collector Systems, Focussed towards the Design of a New Conductor Rail. Vehicle System Dynamics. In process of publication as of May 2005.

2 RID - Reglamento Relativo al Transporte Internacional por Ferrocarril de Mercancías Peligrosas (Regulations concerning the International Carriage of Dangerous Goods by Rail, in Spanish). Ministerio de Fomento. Madrid 2003.

3 Stichel, S. How to Improve the Running Behavior of Freight Wagons with UIC-Link Suspension. Vehicle System Dynamics, Supplement 33, pp. 394-405, 1999.

4 Iwnicki, S. The Manchester Benchmarks for Rail Vehicle Simulation. Manchester Metropolitan University - Rail Technology Unit, 1998

5 Salem, M. I., Mucino, V. H., Gautam, M. and Aquaro, M. Review of Parameters Affecting Stability of Partially Filled Heavy-Duty Tankers. Society of Automotive Engineers (SAE), Technical Paper No. 1999-01-3709, 1999.

6 Abramson, H. N. The Dynamic Behavior of Liquids in Moving Containers - With Applications to Space Vehicle Technology. NASA SP-106, National Aeronautics and Space Administration, 1966.

7 Ranganathan, R., Ying, Y. and Miles, J. B. Analysis of Fluid Slosh in Partially Filled Tanks and Their Impact on the Directional Response of Tank Vehicles. Society of Automotive Engineers (SAE), Technical Paper No. 932942, 1993.

8 Ranganathan, R., Ying, Y. and Miles, J. B. Development of a Mechanical Analogy Model to Predict the Dynamic Behavior of Liquids in Partially Filled Tank Vehicles. Society of Automotive Engineers (SAE), Technical Paper No. 942307, 1994.

9 Bogomaz, G. I., Markova, O. M. and Chernomashentseva, YU. G. Mathematical Modelling of Vibrations and Loading of Railway Tank Taking into Account the Liquid Cargo Mobility. Vehicle System Dynamics, 30, pp. 285-394, 1998.

10 Aliabadi, S., Johnson, A. and Abedi, J. Comparison of Finite Element and Pendulum Models for Simulation of Sloshing. Computer \& Fluids, Vol. 32, pp. 535-545, 2003.

11 Budiansky, B. Sloshing of Liquids in Circular Canals and Spherical Tanks. Journal of the Aero/Space Sciences, Vol. 27, No. 3, 1960.

12 prEN 14363 Railway Applications - Testing for the Acceptance of Running Characteristics of Railway Vehicles - Testing of Running Behaviour and Stationary Tests. September 2002.

13 UIC 518 Testing and Approval of Railway Vehicles from the Point of View of Their Dynamic Behavior - Safety - Track Fatigue - Ride Quality. 2nd edition, April 2003.

14 Lamb, H. Hydrodynamics. $6^{\text {th }}$ edition, Dover. New York 1945. 